\title{
Finally, A Tool to Address Extubation Anxiety!
}

\author{
Girija G. Konduri ${ }^{1}$ Joanne Lagatta ${ }^{1}$ Satyan Lakshminrusimha ${ }^{2} \cdot$ Ola Didrik Saugstad $^{3,4}$
}

Received: 11 September 2019 / Revised: 23 September 2019 / Accepted: 30 September 2019 / Published online: 17 October 2019

(c) Springer Nature America, Inc. 2019

Extremely premature infants ( $<28$ weeks gestation at birth) have a significant risk of needing invasive mechanical ventilation and developing bronchopulmonary dysplasia (BPD) as a complication. While they account for $0.5-1 \%$ of live births, they represent a disproportionate share of infants in the Neonatal intensive care units (NICU) due to their prolonged NICU stay [1, 2]. The increasing survival of these infants in recent times was facilitated by rapid advances in neonatal intensive care [3]. However, these infants remain at the highest risk for long-term pulmonary and neuro-developmental impairments (NDI) among all births.

Invasive mechanical ventilation facilitates survival of these infants; yet prolonged mechanical ventilation is associated with adverse consequences, including BPD and NDI [4]. Early extubation to less invasive modalities, such as CPAP or nasal intermittent mandatory ventilation are logical strategies to decrease barotrauma/volutrauma, airway injury and risk of nosocomial infections. Indeed, some studies have found that the total duration of invasive mechanical ventilation is a strong predictor of both BPD and NDI [4]. However, extubation too early before the infant is ready, can lead to a period of respiratory instability with recurrent hypoxemia and acidosis and reintubation procedure with the attendant risks. Extubation failure is related to immaturity of the respiratory control center, structural immaturity of lung

Girija G. Konduri

gkonduri@mcw.edu

1 Department of Pediatrics, Medical College of Wisconsin and Children's Research Institute, Children's Hospital of Wisconsin, Milwaukee, WI, USA

2 Department of Pediatrics, University of California-Davis, Sacramento, CA, USA

3 Department of Pediatric Research, University of Oslo, Oslo, Norway

4 Ann and Robert Lurie Children's Hospital of Chicago and Northwestern University Feinberg School of Medicine, Chicago, IL, USA parenchyma and airways, surfactant deficiency, patent ductus arteriosus with left to right shunt, excessive compliance of the chest wall, acquired airway abnormalities and intraventricular hemorrhage (Fig. 1).

Estimating the readiness of a premature infant for extubation is a complex decision process that requires incorporating several clinical factors. Failure of extubation can lead to reintubation with the procedural risks and often higher ventilator support than prior to extubation. The additional risk imposed on these infants by failed extubation has been studied previously and is the subject of ongoing investigations [5-7]. A previous study by Shalish et al. demonstrated that failed extubation is associated with an increased risk of death/moderate-severe BPD, even after adjusting for the cumulative duration of ventilation [5]. Reintubation within $48 \mathrm{~h}$ was associated with a particularly increased risk of death/BPD [5]. Infants with extubation failure were younger and smaller at the time of birth and at extubation in this study [5]. An increased risk of death/BPD and longer duration of ventilator support was also reported after failed extubation by Manley et al. from the New Zealand clinical trials network in a group of 174 extremely preterm infants [6]. Extubation failure was defined as reintubation within 7 days; higher gestational age and lower pre-extubation $\mathrm{PaCO}_{2}$ were associated with extubation success [6]. In contrast, other studies suggest that a decrease in total duration of mechanical ventilation lowers the risk of BPD, despite episodes of reintubations [4]. Weighing the risks of early extubation vs. the potential benefits of shortening the duration of invasive ventilation remains a challenging dilemma for the clinician at the bedside. Developing objective criteria that predict the success/failure rates of extubation can be a valuable bedside clinical tool. Bedside readiness tests, including assessment of spontaneous breathing effort have not been validated systematically to be recommended at this point [7].

In a study published in this issue of Journal of Perinatology, Gupta et al. report on an online calculator to help assess the chances of extubation success for a group of extremely preterm infants [8]. The study is based on a 
Fig. 1 Factors associated with extubation failure in extremely preterm infants. Immature respiratory drive, airway abnormalities, immature lungs stiff from surfactant deficiency, inflammation or fibrosis, pliable rib cage and chest wall, and pulmonary over-circulation with edema from a left to right shunt such as patent ductus arteriosus (PDA) contribute to extubation failure. (Copyright Girija G. Konduri and Satyan Lakshminrusimha)

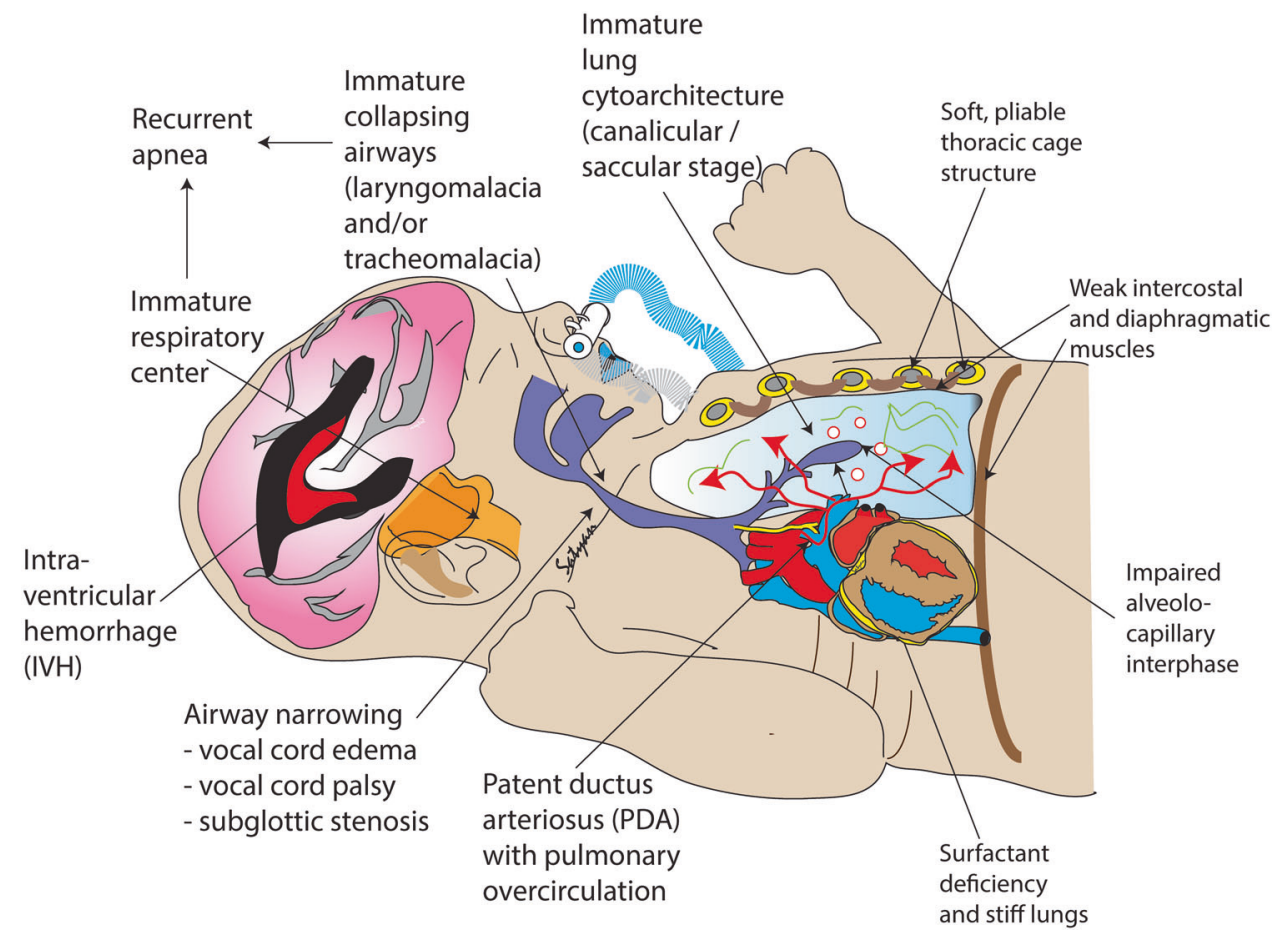

retrospective analysis of data from a single center with evaluation of extubation outcomes for preterm infants with birth weight $<1250 \mathrm{~g}$, during the first 60 days of life. The primary outcome was extubation success, defined as surviving for at least 5 days after extubation, without a need for reintubation. They report that $73 \%$ of infants had successful extubation; these infants were larger, born at older gestation and had higher preextubation $\mathrm{pH}$ and lower respiratory severity score in the first $6 \mathrm{~h}$ of age than those who failed, factors that they incorporated into the online tool. Strengths of this study include the relatively large number of subjects, bootstrapping techniques to obtain confidence intervals, and the similarity of their cohort's extubation success and definitions to other previous studies on this topic. The time window selected for inclusion of reintubation attempts is consistent with other reports that majority of respiratory related reintubations occur within 7 days of extubation [9]. The addition of an online calculator offers the potential for bedside applicability of these findings. The online tool link is included only for the reader's convenience and not specifically endorsed by this Journal or its editorial staff.

The main limitations of the study relate to this being a single center, retrospective study with limited detail on the circumstances surrounding care for these intubated infants. Details of the institution's extubation/reintubation criteria, adverse events associated with extubation failure, and blood gas or other data preceding reintubation are needed to interpret the results. It is unclear whether some extubation failures were potentially "worse" than others. Single-center data may not be generalizable to centers with different practices for early mechanical ventilation versus noninvasive positive pressure support. The success of extubation is highly dependent on whether infants were intubated in the delivery room, using Intubation Surfactant Extubation or Less Invasive Surfactant Instillation technique. The criteria for extubation and reintubation were not standardized in the present study and left to the discretion of clinicians. A few eligible infants were excluded since they were enrolled in an ongoing multi-center prospective evaluation of extubation readiness $[5,8]$; the upcoming results of such study may potentially address some limitations pointed out for the present study. The variables in the model are treated as though they are associated with the outcome in a linear fashion; specific cut-points may be more useful for bedside decision making. This online tool also raises some questions, such as whether infants with a lower predicted probability of success "fail" differently, more quickly, or with more consequences? Does a lower calculated probability of success create a self-fulfilling prophecy where there are fewer attempts for infants who could potentially be extubated successfully?

Despite these limitations, calculator-style online tools are widely utilized and likely to be of much clinical interest. Perhaps the most valuable aspect of the bedside clinical tool is the recognition of various clinical factors that can influence the bedside decision rather than the absolute number presented by the calculator. For example, using the tool to predict the extubation success for a 24-week gestation 
premature infant who weighs $750 \mathrm{~g}$ on day of life five with pre-extubation $\mathrm{pH}$ of 7.25 , requiring $25 \% \mathrm{O}_{2}$ and the highest respiratory severity score in first $6 \mathrm{~h}$ of 4 has a predicted success score of $34 \%$. Rather than relying on this single number, recognizing the various clinical variables presented in the calculator would aid the clinicians better in making a more informed decision. In future, a prospective study that defines the criteria a priori for extubation/reintubation and documents associated adverse events would provide additional clarity for this clinical decision. While the results of such study are awaited, the components of the calculator can provide an important checklist for the clinician at the bedside making this decision. The results of the present report also serve as an important aid during the discussion with parents about the likelihood of success and need for further intervention at the time of this clinical decision. In future, strategies to decrease extubation failure, such as the use of synchronized nasal intermittent mandatory ventilation, need to be tested in randomized controlled trials.

Funding Supported by grants 1 R01 HL136597-01 from NHLBI and Muma Endowed Chair of Neonatology from Children's Research Institute of Children's Hospital of WI (GGK), K23HL136525 from NHLBI (JL), and 5 R01 HD072929 (SL).

Author contributions GGK and JL prepared the draft. SL and ODS have edited the manuscript and added comments. GGK finalized the manuscript.

\section{Compliance with ethical standards}

Conflict of interest The authors declare that they have no conflict of interest.
Publisher's note Springer Nature remains neutral with regard to jurisdictional claims in published maps and institutional affiliations.

\section{References}

1. Anderson JG, Baer RJ, Partridge JC, Kuppermann M, Franck LS, Rand L et al. Survival and major morbidity of extremely preterm infants: a population-based study. Pediatrics. 2016;138.

2. Stoll BJ, Hansen NI, Bell EF, Shankaran S, Laptook AR, Walsh $\mathrm{MC}$, et al. Neonatal outcomes of extremely preterm infants from the NICHD neonatal research network. Pediatrics. 2010;126:443-56.

3. Costeloe KL, Hennessy EM, Haider S, Stacey F, Marlow N, Draper ES. Short term outcomes after extreme preterm birth in England: comparison of two birth cohorts in 1995 and 2006 (the EPICure studies). Brit Med J. 2012;345:e7976.

4. Jensen EA, DeMauro SB, Kornhauser M, Aghai ZH, Greenspan JS, Dysart KC. Effects of multiple ventilation courses and duration of mechanical ventilation on respiratory outcomes in extremely low-birthweight infants. JAMA Pediatr. 2015;169:1011-7.

5. Shalish W, Kanbar L, Kovacs L, Chawla S, Keszler M, Rao S, et al. The impact of time interval between extubation and reintubation on death or bronchopulmonary dysplasia in extremely preterm infants. J Pediatr. 2019;205:70-76.e2.

6. Manley BJ, Doyle LW, Owen LS, Davis PG. Extubating extremely preterm infants: predictors of success and outcomes following failure. J Pediatr. 2016;173:45-9.

7. Shalish W, Latremouille S, Papenburg J, Sant'Anna GM. Predictors of extubation readiness in preterm infants: a systematic review and meta-analysis. Arch Dis Child Fetal Neonatal Ed. 2019;104: F89-97.

8. Gupta D, Greenberg RG, Sharma A, Natarajan G, Cotton M, Thomas R. et al. A predictive model for extubation readiness in extremely preterm infants. J Perinatol. https://doi.org/10.1038/ s41372-019-0475-x.

9. Shalish W, Kanbar L, Keszler M, Chawla S, Kovacs L, Rao S, et al. Patterns of reintubation in extremely preterm infants: a longitudinal cohort study. Pediatr Res. 2018;83:969-75. 